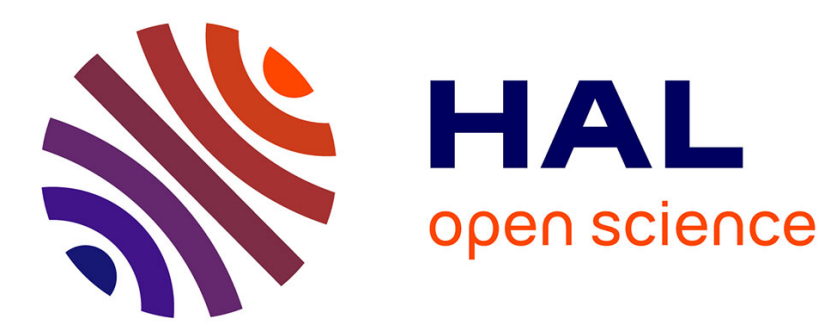

\title{
Étude des profils de raies d'émission d'un plasma d'arc stabilisé à symétrie cylindrique.
}

\author{
J.-L. Queffelec, M. Girault
}

\section{To cite this version:}

J.-L. Queffelec, M. Girault. Étude des profils de raies d'émission d'un plasma d'arc stabilisé à symétrie cylindrique.. Revue de Physique Appliquée, 1971, 6 (3), pp.401-407. 10.1051/rphysap:0197100603040100 . jpa-00243562

\section{HAL Id: jpa-00243562 https://hal.science/jpa-00243562}

Submitted on 1 Jan 1971

HAL is a multi-disciplinary open access archive for the deposit and dissemination of scientific research documents, whether they are published or not. The documents may come from teaching and research institutions in France or abroad, or from public or private research centers.
L'archive ouverte pluridisciplinaire HAL, est destinée au dépôt et à la diffusion de documents scientifiques de niveau recherche, publiés ou non, émanant des établissements d'enseignement et de recherche français ou étrangers, des laboratoires publics ou privés. 


\title{
ÉTUDE DES PROFILS DE RAIES D'ÉMISSION D'UN PLASMA D'ARC STABILISÉ A SYMÉTRIE CYLINDRIQUE.
}

\author{
J.-L. QUEFFELEC et M. GIRAULT
}

Laboratoire de Physique Moléculaire, Faculté des Sciences, Rennes

(Reçu le 28 janvier 1971, révisé le 28 avril 1971)

\begin{abstract}
Résumé. - Nous décrivons deux sources $S_{1}$ et $S_{2}$ de plasma d'arc stabilisé à la pression atmosphérique, dans de l'argon et un mélange d'argon et d'hydrogène. La symétrie du plasma est cylindrique. La température le long de l'axe de $S_{2}$ est constante à $1 \%$ près. Les techniques de spectrométrie photoélectrique par enregistrement mises au point permettent l'étude de l'élargissement, du déplacement et de la forme des raies de l'argon en fonction de la température, par observation transversale ou axiale du plasma.
\end{abstract}

Abstract. - Two stabilized arc plasma sources $\mathbf{S}_{1}$ and $\mathbf{S}_{2}$ at atmospheric pressure, in argon and a mixure of argon and hydrogen are described. The plasma has a cylindrical symmetry. The axial temperature of the $S_{2}$ plasma source is constant within $1 \%$. The checked up recording photoelectric spectrometry technics, allow us to study widths, shifts and shapes of argon lines versus temperature, by observing the plasma side on or end on.

1. Introduction. - Le premier arc stabilisé mis au point $\left(S_{1}\right)$ [1], fonctionnant à la pression atmosphérique, nous a permis de nous initier à la production de plasmas stables à symétrie cylindrique, dans l'argon et dans des mélanges d'argon et d'hydrogène, et de mettre au point quelques techniques aboutissant au tracé des profils des raies émises par des éléments du plasma situés à différentes distances de l'axe, par spectroscopie photoélectrique dans le visible. Ces techniques, propres à l'observation latérale de plasmas à symétrie cylindrique, nous ont conduit, d'une part, à déterminer la distribution radiale des températures dans un disque de plasma grâce à la méthode de l'élargissement par effet Stark de la raie $\mathrm{H}_{\beta}$ de l'hydrogène, et, d'autre part, à mesurer la demi-largeur et la position de la raie $4300 \AA$ de l'Ar I à différentes distances de l'axe ; ceci nous a permis d'étudier la demi-largeur et le déplacement en fonction de la température.

Après ces premières mesures, et pour étudier les profils des raies d'émission du plasma en fonction de la température, nous avons mis au point un second arc stabilisé $\left(S_{2}\right)$ dont la température le long de l'axe est constante à un pour cent près. L'observation axiale de cette source de plasma nous permet ainsi d'enregistrer directement les profils des raies d'émission à température uniforme : la température axiale, reliée directement à la demi-largeur de la raie $\mathrm{H}_{\beta}$ sur l'enregistrement.

Nous commencerons par décrire les sources de plasma $S_{1}$ et $S_{2}(\S 2)$ puis le montage de spectroscopie photoélectrique et les techniques d'enregistrement des profils de raies $(\S 3)$. Nous préciserons les perfor- mances de chaque technique et donnerons des résultats de mesures d'élargissement et de déplacement de la raie $4300 \AA$ de l'Ar I. Puis, en étudiant la source $S_{2}$ (§ 4) après avoir vérifié que la température est uniforme le long de l'axe, nous montrerons que l'observation axiale permet l'étude précise de la forme des profils de raies, de leur élargissement et de leur déplacement en fonction de la température.

2. Sources de plasma. - 2.1 Principe. - Les deux arcs stabilisés $S_{1}$ et $S_{2}$ mis au point sont du type Maecker [2]. La stabilisation du plasma est obtenue en contraignant la flamme de l'arc à passer à travers les orifices centraux $(\varphi=5 \mathrm{~mm})$ d'une série de disques en cuivre coaxiaux refroidis par une circulation d'eau et séparés par des rondelles isolantes en bakélite.

2.2 Source $\mathrm{S}_{1}$. - Le schéma de principe est donné par la figure 1. Le gaz plasmagène (Ar ou

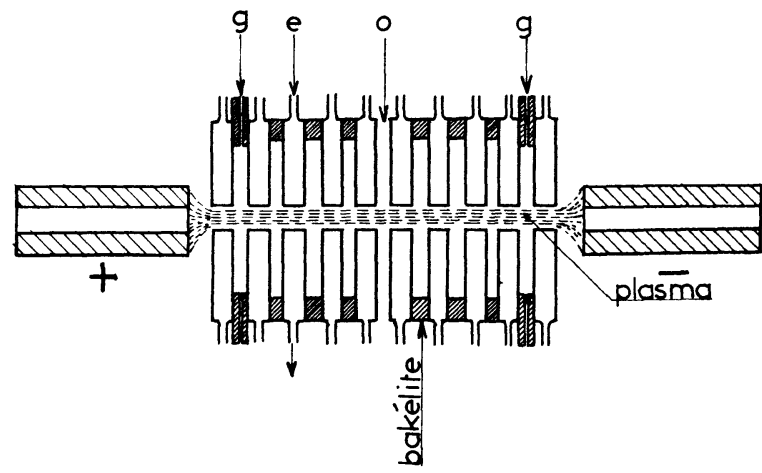

Fig. 1. - Schéma de principe de l'arc $\mathrm{S}_{1} . e$ : refroidissement par circulation d'eau des disques en cuivre. $g$ : admission du gaz plasmagène. $o$ : observation. 
mélange $\mathrm{Ar}+\mathrm{H}_{2}$ ) est introduit dans l'arc par l'intermédiaire de deux disques spéciaux $(g)$ percés de canaux débouchant tangentiellement aux parois intérieures afin d'obtenir un courant gazeux homogène et d'éviter le soufflage de l'arc. Les électrodes creuses ( $\varphi$ int $=7 \mathrm{~mm})$ en graphite dont les supports sont refroidis par une circulation d'eau, sont refroidies elles-mêmes intérieurement par un courant d'Ar pur : ainsi, on réduit beaucoup leur vitesse d'usure; on obtient un plasma très stable. L'observation du plasma se fait latéralement $(\mathrm{O})$ entre deux disques en cuivre dans la région centrale de l'arc.

2.3 Source $\mathrm{S}_{2}$. - Cet arc, dont le schéma de principe est donné par la figure 2, se rapproche, dans sa conception, de ceux décrits par d'autres auteurs [3], [4]. Du fait que nous étudions des gaz différents, des modifications ont été nécessaires et concernent particulièrement: le diamètre du canal de confinement du plasma et la disposition des arrivées et des sorties de gaz plasmagène. La possibilité d'observation latérale de la colonne de l'arc en trois régions distinctes constitue une des originalités de notre réalisation.

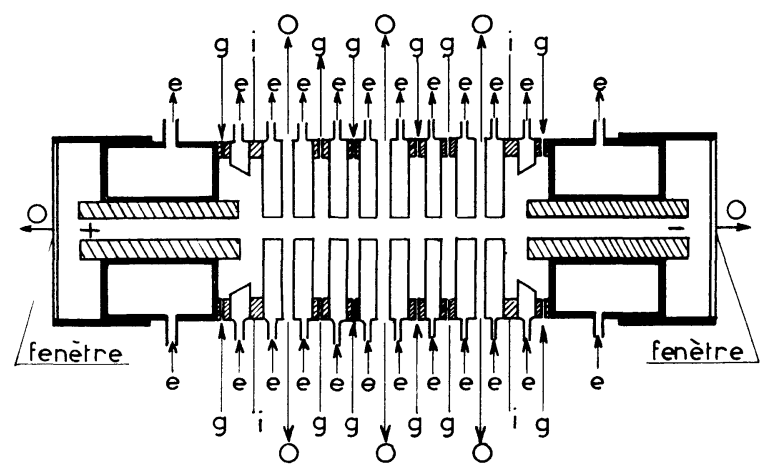

Fig. 2. - Schéma de principe de l'arc $S_{2}$. $e$ : entrées et sorties de l'eau de refroidissement des disques en cuivre et des supports d'électrodes. $g$ : entrée et sortie du gaz plasmagène. $i$ : isolant. $O$ : fenêtres d'observation axiale et latérale.

Le gaz est ici admis tangentiellement à la colonne de l'arc par l'intermédiaire de quatre disques spéciaux $(g)$. Les deux arrivées extrêmes assurent un refroidissement supplémentaire des électrodes. La sortie des gaz se fait radialement par des disques spéciaux. En fonctionnement, la tache cathodique se localise en une petite région fixe de l'électrode, contribuant ainsi à rendre l'arc très stable pendant plus d'une heure.

L'observation du plasma peut se faire suivant l'axe ou par l'une des trois ouvertures latérales (O).

2.4 Alimentation ÉlectriQue DE L'ARC. - L'alimentation électrique est assurée par un redresseur que nous avons réalisé [2] à partir des différents éléments. La figure 3 donne le schéma du montage: on redresse les deux alternances de chacune des trois phases du secteur. Les diodes (D) de puissance au silicium (24 R 4 Thomson-Houston), sont montées sur ailettes en cuivre, en ventilation naturelle. Les résistances (R) utilisées sont de $4700 \Omega$ et les capacités (C) de $1000 \mathrm{pF}$. Ce redresseur peut fournir $200 \mathrm{~A}$ sous $300 \mathrm{~V}$ environ. En charge, le taux d'ondulation de la tension redressée est de 0,011 , ce qui correspond à un facteur de forme très voisin de l'unité.

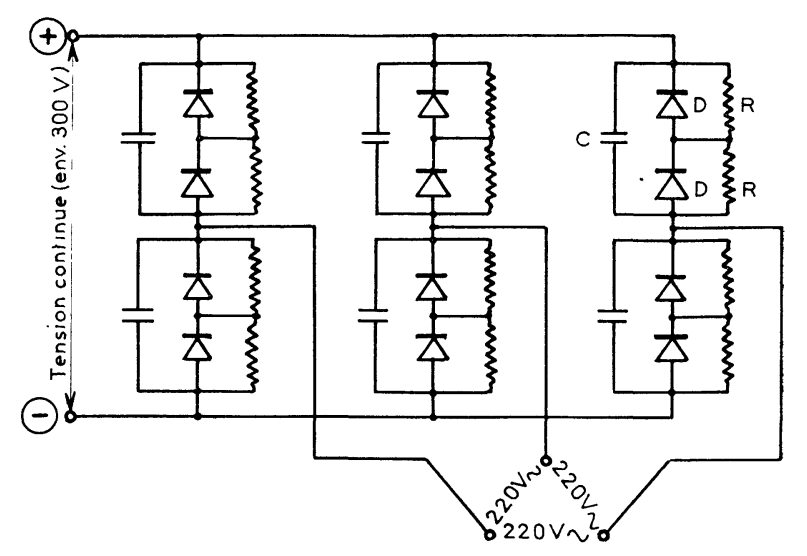

FIG. 3. - Schéma du redresseur.

Pour les courants d'arcs que nous utilisons, la chute de tension aux bornes de l'arc est bien inférieure à la tension fournie par l'alimentation. Nous avons donc réduit la tension fournie par le redresseur à l'aide d'une résistance de valeur maximum $5 \Omega$, variable par plots, placée en série avec l'arc.

Cette résistance permet de faire varier le courant d'arc de 40 à 150 A environ et augmente la stabilité de la source.

\section{Mesure de la distribution radiale des températures} d'un disque de plasma à symétrie cylindrique. 3.1 ChOIX DE LA MÉTHODE. - Plusieurs méthodes de détermination de température d'un plasma ont jusqu'ici été mises au point [5], [6]. Certains auteurs [7] ont fait une étude comparée de ces méthodes; ils ont montré que celle qui est basée sur l'élargissement par effet Stark de la raie $\mathrm{H}_{\beta}$ de l'hydrogène donne les mesures les plus précises de la concentration électronique du plasma et de sa température. En outre, au cas où les plasmas sont stables et exempts de fluctuations importantes, ce qui est le cas pour notre plasma, la spectrophotométrie photoélectrique par enregistrement est la technique qui donne les meilleurs résultats expérimentaux. Cette méthode et cette technique que nous avons choisies d'appliquer, nous ont conduit à utiliser comme gaz plasmagène de l'argon avec un faible pourcentage d'hydrogène $(3 \%)$.

3.2 Etude théorique - Les travaux de Griem [8] sur l'élargissement par effet Stark de la raie $\mathrm{H}_{\beta}$ de l'hydrogène donnent la demi-largeur théorique $\Delta \lambda$ de $\mathrm{H}_{b}$ en fonction de la concentration électronique $N_{\mathrm{e}}$ du plasma pour diverses températures; dans le domaine des températures explorées dans ce travail $\left(8000^{\circ} \mathrm{K}<T<17000^{\circ} \mathrm{K}\right.$ ), pour lequel $N_{\mathrm{e}}$ est supé- 
rieur à $10^{15}$ électrons $/ \mathrm{cm}^{3}$, on peut admettre, d'après Griem, que $\Delta \lambda$ est seulement fonction de $N_{\mathrm{e}}$. Dans un plasma donné, $N_{\mathrm{e}}$ est fonction de $T: N_{\mathrm{e}}=f(T)$ (soit $T^{\circ} \mathrm{K}=G\left(N_{\mathrm{e}}\right)$ ). Les mesures permettent de déterminer $\Delta \lambda$ de $\mathrm{H}_{\beta}$ en chaque point du plasma. De cette valeur de $\Delta \lambda$ et par comparaison avec l'expression théorique $N_{\mathrm{e}}=f(\Delta \lambda)$, on déduit la concentration électronique $N_{\mathrm{e}}$; de cette concentration, en s'aidant de la courbe $T^{\mathrm{o}} \mathrm{K}=G\left(N_{\mathrm{e}}\right)$, on déduit la température.

La fonction $N_{\mathrm{e}}=f(T)$ doit donc être déterminée pour le plasma considéré.

Un plasma à la pression atmosphérique dont la température est inférieure à $16 \times 10^{3}{ }^{\circ} \mathrm{K}$ est en équilibre thermodynamique ; il est quasiment neutre et ne contient que des atomes neutres, des atomes une fois ionisés et des électrons [8].

En désignant par $N_{\mathrm{A}}^{0}, N_{\mathrm{A}}^{+}, N_{\mathrm{H}}^{0}, N_{\mathrm{H}}^{+}$et $N_{\mathrm{e}}$ les concentrations respectives, dans le plasma, des atomes d'argon neutres et une fois ionisés, des atomes d'hydrogène neutres et une fois ionisés et des électrons, nous pouvons écrire les équations du plasma.

$$
\begin{aligned}
& \frac{N_{\mathrm{A}}^{+} N_{\mathrm{e}}}{N_{\mathrm{A}}^{0}}=\left(\frac{2 \pi m k T}{h^{2}}\right)^{3 / 2} 2 \frac{U_{\mathrm{A}}^{+}(T, \Delta E)}{U_{\mathrm{A}}^{0}(T, \Delta E)} \times \\
& \times \exp \left[-\frac{E_{\mathrm{A}}^{0}-\Delta E}{k T}\right] \\
& \frac{N_{\mathrm{H}}^{+} N_{\mathrm{e}}}{N_{\mathrm{H}}^{0}}=\left(\frac{2 \pi m k T}{h^{2}}\right)^{3 / 2} 2 \frac{U_{\mathrm{H}}^{+}(T, \Delta E)}{U_{\mathrm{H}}^{0}(T, \Delta E)} \times \\
& \times \exp \left[-\frac{E_{\mathrm{H}}^{0}-\Delta E}{k T}\right] \\
& x=\frac{N_{\mathrm{H}}^{+}+N_{\mathrm{H}}^{0}}{N_{\mathrm{A}}^{+}+N_{\mathrm{A}}^{0}} \\
& P=\left(N_{\mathrm{A}}^{0}+N_{\mathrm{A}}^{+}+N_{\mathrm{H}}^{0}+N_{\mathrm{H}}^{+}+N_{\mathrm{e}}\right) k T \\
& N_{\mathrm{e}}=N_{\mathrm{A}}^{+}+N_{\mathrm{H}}^{+} \text {, }
\end{aligned}
$$

où (1) et (2) sont les équations de Saha-Eggert [9] [10], relatives à l'argon et à l'hydrogène.

$E_{\mathrm{A}}^{0}$ et $E_{\mathrm{H}}^{0}$ sont les énergies d'ionisation des atomes neutres d'argon et d'hydrogène pour une concentration électronique nulle; $\Delta E$ a pour valeur $2 \mathrm{e}^{3}$ $\left(2 \pi N_{\mathrm{e}} / k T\right)$ [11] [12].

$U_{\mathrm{A}}^{0}, U_{\mathrm{A}}^{+}, U_{\mathrm{H}}^{0}$ et $U_{\mathrm{H}}^{+}$sont les fonctions de partition des atomes d'argon et d'hydrogène neutres et une fois ionisés. Les tables de Drawin et Felenbok [13] donnent ces fonctions pour différentes valeurs de $T$ et de $\Delta E$.
(3) est le rapport des nombres d'atomes initiaux d'argon et d'hydrogène dans le mélange.

(4) est l'équation des gaz parfaits.

(5) exprime la quasi-neutralité.

Nous avons programmé sur une machine « I. B. M. 1620 » la résolution de ce système de cinq équations à cinq inconnues $\left(N_{\mathrm{A}}^{0}, N_{\mathrm{A}}^{+}, N_{\mathrm{H}}^{0}, N_{\mathrm{H}}^{+}\right.$et $\left.N_{\mathrm{e}}\right)$; nous avons fait appel à la méthode itérative de Newton [14], et plus particulièrement à la méthode dite de la sécante, qui permet de résoudre des équations de la forme $f(x)=0$, soit ici $f\left(N_{\mathrm{e}}\right)=0$, pour une température donnée.

Les résultats $\mathrm{du}$ calcul sont présentés dans le tableau ci-dessous pour $x=3 / 97$ et $p=760$ torr.

3.3 Etude expérimentale. Source $S_{1}$. - Le travail expérimental consiste à tracer les profils des raies d'émission du plasma en fonction de la distance à l'axe $r_{k}$. En ce qui concerne la raie $\mathrm{H}_{\beta}$, sa demi-largeur $\Delta \lambda_{r_{\kappa}}$ nous donnera la température.

Le montage de spectroscopie photoélectrique comprend un spectrographe Jarrell Ash stigmatique à réseau tournant (dispersion constante de 2,55 $\AA / \mathrm{mm}$ dans le premier ordre) transformé en monochromateur. $\mathrm{La}$ réponse d'un photomultiplicateur du type « 53 A. V.P. » de la « Radio Technique » est transmise à un enregistreur Honeywell « Electronik 19 ». Avec ce montage, nous avons mis au point deux techniques d'enregistrement des raies d'émission du plasma.

3.3.1 Première technique. - L'axe de l'arc est perpendiculaire à la fente d'entrée du spectrographe sur laquelle on forme l'image d'un disque de plasma au moyen d'une lentille. Immédiatement devant la fente, un cache, muni d'une fente de largeur $0,4 \mathrm{~mm}$ inclinée à $45^{\circ}$ peut coulisser et permet ainsi, par déplacements successifs, de sélectionner un élément $\Delta x$ correspondant à une colonne de plasma située à une distance $x_{i}$ de l'axe; la rotation du réseau permet d'enregistrer le profil $I_{x_{i}}(\lambda)$ de la raie $\mathrm{H}_{\beta}$ correspondant à $x_{i}$.

La fente d'entrée a une longueur totale de $20 \mathrm{~mm}$ et nous n'en utilisons que la partie centrale $(10 \mathrm{~mm})$ pour obtenir nos enregistrements. Une lentille de champ est disposée devant la fente d'entrée; les dimensions respectives du miroir sphérique du spectrographe $(\varphi=40 \mathrm{~cm})$ et de la partie striée du réseau plan $(10 \mathrm{~cm} \times 5,5 \mathrm{~cm})$ sont telles que cette dernière reste toujours complètement couverte par le faisceau, quelle que soit la partie utilisée de la fente d'entrée : le faisceau utilisé est donc constamment diaphragmé par le réseau.

$\begin{array}{ccccccc}T\left({ }^{\circ} \mathrm{K}\right) & 7000 & 8000 & 9000 & 10000 & 11000 & 12000 \\ \overline{N_{\mathrm{e}}} & 2,84 \times 10^{14} & 1,48 \times 10^{15} & 5,39 \times 10^{15} & 1,51 \times 10^{16} & 3,44 \times 10^{16} & 6,6 \times 10^{16} \\ T\left({ }^{\circ} \mathrm{K}\right) & 13000 & 14000 & 15000 & 16000 & 17000 & - \\ N_{\mathrm{e}} & 1,08 \times 10^{17} & 1,5 \times 10^{17} & 1,82 \times 10^{17} & 1,98 \times 10^{17} & 2,01 \times 10^{17} & -\end{array}$


Les profils $I_{x_{i}}(\lambda)$ étant enregistrés, il est ensuite nécessaire de passer des profils $I_{x_{i}}(\lambda)$ aux profils $I_{r_{k}}(\lambda)$ relatifs à un élément de volume du plasma situé à la distance $r_{k}$ de l'axe. A cet effet, on utilise, entre autres procédés de calcul, la transformation d'Abel. Chacune des courbes $I_{r_{k}}(\lambda)$ permet de déterminer la demi-largeur expérimentale $\Delta \lambda_{r_{k}}$ de la raie $\mathrm{H}_{\beta}$.

Nous nous sommes assurés de la reproductibilité des résultats. Vingt minutes sont nécessaires pour effectuer l'ensemble des enregistrements $I_{x_{i}}(\lambda)$ correspondant à la mesure de la distribution radiale des températures. Nous avons enregistré cinq profils correspondant à la même valeur de $x_{i}$ à $5 \mathrm{~mm}$ d'intervalle : les variations en intensité des profils sont inférieures à $2 \%$, la demi-largeur restant constante à $0,5 \%$ près.

Des mesures faites par Shumaker et Wiese [7] sur des plasmas de composition voisine de celle que nous utilisons, ont montré que le coefficient de selfabsorption au centre de la raie $\mathrm{H}_{\alpha}$ est inférieur à $1 \%$ et bien inférieur encore pour les autres raies de la série de Balmer. Nous avons donc considéré que le plasma est optiquement mince pour la raie $\mathrm{H}_{\beta}$.

La courbe de distribution radiale des températures $T=f(r)$ de la figure 4, correspond à une intensité de courant d'arc de 40 A. En tenant compte de la légère surpression à l'intérieur de l'arc [15] et des incertitudes commises sur les lectures et les transformations de courbes, nous calculons que l'erreur relative d'ordre expérimental sur la mesure de la température axiale est inférieure à $5 \%$.

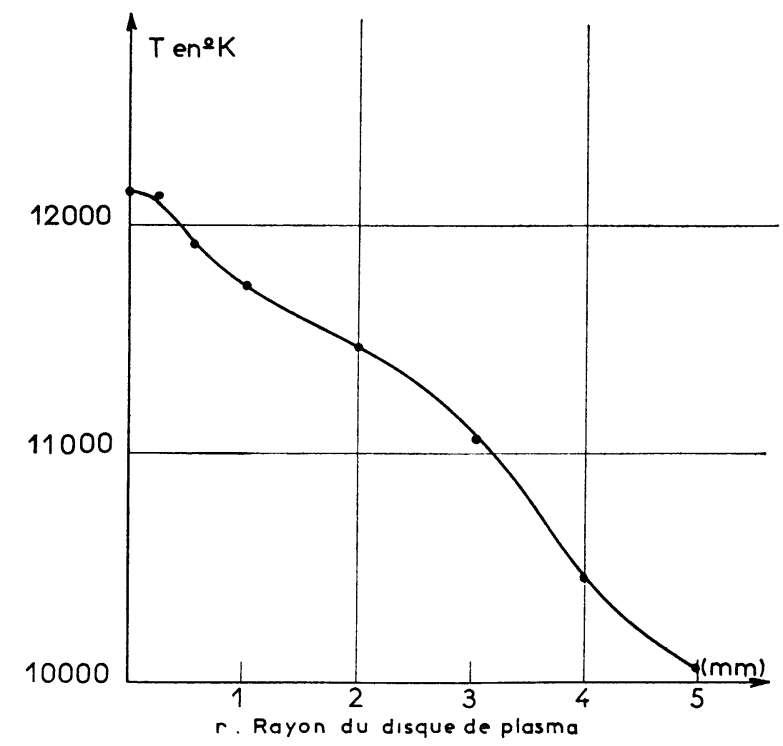

FIG. 4. - Distribution radiale des températures.

Notons que, dans le plasma, les raies d'émission de l'Ar I et de l'Ar II subissent un élargissement par effet Stark et un déplacement, tous deux fonctions de la température. Si aucune longueur d'onde n'est repérée sur les profils $I_{x_{i}}(\lambda)$, cette technique d'enregistrement ne peut être utilisée pour obtenir les profils $I_{r_{k}}(\lambda)$ des raies de l'Ar.
3.3.2 Deuxième technique. - 3.3.2.1 Principe. Comme l'indique la figure 5 , on forme une image intermédiaire d'un disque vertical de plasma sur l'une des extrémités du diamètre horizontal d'un disque métallique tournant à vitesse constante (1 tour en $6 \mathrm{~mm}$ ) autour d'un axe parallèle à l'axe optique du

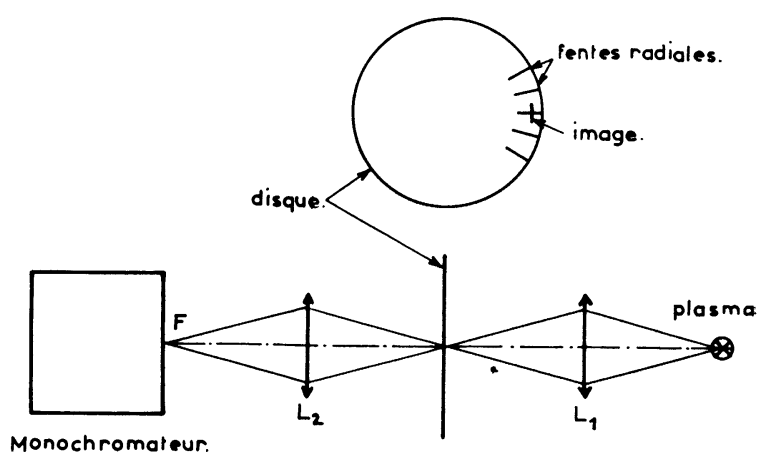

FIG. 5. - Schéma du dispositif expérimental.

système. Ce disque, de $15,5 \mathrm{~cm}$ de rayon, possède 80 fentes radiales (largeur : 0,4 $\mathrm{mm}$ ) à l'extrémité de rayons angulairement équidistants. Comme dans le premier montage, on sélectionne, à l'aide de ce disque, sur la fente d'entrée du spectrographe, un élément $\Delta x$ correspondant à une colonne de plasma située à une distance $x$ de l'axe, mais la rotation simultanée du disque et du réseau nous permet d'enregistrer des profils $I(x, \lambda)$. L'élément de raie $(\Delta \lambda, \Delta x)$ sélectionné se déplace avec des vitesses $V_{x}$ et $V_{\lambda}$ suivant les axes $x$ et $\lambda$ correspondants, dans le cas général où les fentes d'entrée et de sortie sont parallèles. L'inclinaison de la fente de sortie du monochromateur nous permet de faire varier $V_{\lambda}$. Pour une vitesse de réseau donnée et une vitesse de disque constante, on peut calculer l'inclinaison $\theta$ de la fente de sortie telle que $V_{\lambda}=0$. Le montage permet alors, au cours d'un seul passage d'une raie devant la fente de sortie du monochromateur d'enregister plusieurs profils $I_{\lambda_{i}}(x)$ à longueur d'onde constante $\lambda_{i}$. Pour la plus faible vitesse du réseau utilisée $(2,82 \AA / \mathrm{mn})$ et une vitesse de 1 tour en $6 \mathrm{mn}$ du disque, $\theta$ est voisin de $20^{\prime}$ et la différence de longueur d'onde entre deux profils successifs est d'environ $0,21 \AA$.

Dans la première technique (§3.3.1), la transformation d'Abel ne s'applique pas directement aux profils $I_{x_{i}}(\lambda)$ enregistrés; il est nécessaire de passer préalablement aux profils $I_{\lambda_{l}}(x)$. On simplifie ainsi, grâce à notre deuxième technique, les calculs permettant de passer des profils $I_{\lambda_{1}}(x)$ enregistrés aux profils $I_{r_{k}}(\lambda)$, relatifs à un élément de volume du plasma situé à la distance $r_{k}$ de l'axe : la transformation d'Abel s'applique directement aux profils $I_{\lambda_{i}}(x)$.

3.3.2.2 Repérage en longueur d'onde. - Les vitesses du réseau et de l'enregistreur étant constantes, il nous suffit de connaître la longueur d'onde correspondant à un point de l'enregistrement. Ce repérage en longueur d'onde se fait au moyen d'un générateur 
d'impulsion à commande photoélectrique, connecté parallèlement au photomultiplicateur, à l'entrée de l'enregistreur. Le générateur transforme en impulsions brèves, un signal lumineux issu d'une " scie à lumière " circulaire, solidaire du mouvement de rotation du réseau. Chaque impulsion se traduit par un pic et correspond à une longueur d'onde précise qui est mesurée par référence au spectre d'une lampe à argon; la précision de cette mesure est évaluée à $\pm 0,03 \AA$ Å.

Le générateur d'impulsion est conforme au schéma de la figure 6. Une photodiode au Germanium (O. A. P. 12) transforme le signal lumineux en signal électrique. Le transistor $T_{1}$ amplifie le signal jusqu'au niveau du seuil de déclenchement d'un circuit de mise en forme (trigger de Schmitt) que constituent les transistors $T_{3}$ et $T_{4}$. Le rhéostat de $1 \mathrm{k} \Omega$ permet d'ajuster le seuil de déclenchement du trigger. $T_{2}$ et $T_{5}$ sont des adaptateurs d'impédances; le premier influe sur le temps de montée du signal (déclenchement plus franc du trigger). Les signaux recueillis sur le collecteur de $T_{4}$ sont rectangulaires et leur transformation en impulsions se fait par un montage différenciateur constitué comme suit: un condensateur de $0,1 \mu^{\mathrm{F}}$ en série avec le curseur du potentiomètre de $100 \Omega$ et la résistance d'entrée de l'enregistreur connecté à la sortie. Le potentiomètre de $100 \Omega$ règle l'amplitude des impulsions.

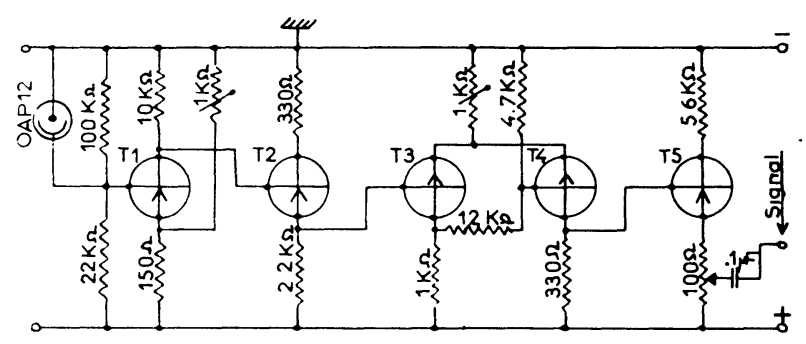

$$
\begin{array}{|l|l:l|}
\hline T 1 & T 2 & A C 125 \\
\hline T 3 & T 4 & B C 109 \\
\hline T 5 & \text { Tension dalimenlation } 9 \mathrm{~V} \\
\hline T S 220 \\
\hline
\end{array}
$$

FIG. 6. - Générateur d'impulsions à commande photoélectrique.

3.3.2.3 Application de la technique. - Contrairement à la première méthode d'enregistrement, cette deuxième technique permet d'obtenir les profils $I_{r_{k}}(\lambda)$ des raies d'émission du plasma qui subissent un élargissement et un déplacement en fonction de la température. La précision du tracé de ces profils sera d'autant plus grande que les raies sont plus larges par rapport à la différence de longueur d'onde entre deux profils successifs $I_{\lambda_{t}}(x)$ sur l'enregistrement. Pour l'étude des raies étroites, les possibilités du montage sont limitées par la plus faible vitesse de rotation du réseau $(2,82 \AA / \mathrm{mm})$ et la plus grande vitesse de déroulement du papier de l'enregistreur $(2,54 \mathrm{~cm} / \mathrm{s})$ pour une vitesse de rotation donnée du disque de la figure 5 .

Dans le cas de notre plasma, nous sommes pratiquement limités à l'étude des raies de l'Ar I. Pour une intensité du courant d'arc de $59 \mathrm{~A}$, nous avons déterminé la distribution radiale des températures et estimé à $3 \%$ la valeur de l'erreur relative expérimentale commise sur la température axiale. Nous avons alors étudié l'élargissement et le déplacement de la raie $4300 \AA$ de l'Ar I. Sur les figures 7 et 8 donnant les valeurs expérimentales (points) de la demi-largeur à mi-hauteur $\Delta \lambda$ et le déplacement $d$ de la raie, nous avons tracé les courbes théoriques (en trait continu) calculées à partir des données de Griem [8].

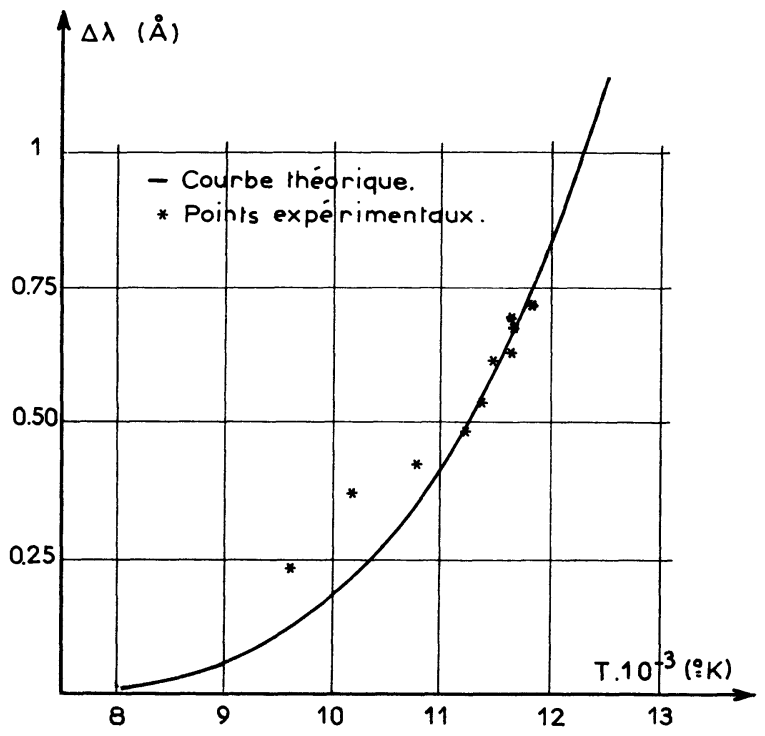

Fig. 7. - Demi-largeur à mi-hauteur de la raie $4300 \AA$ de Ar I.

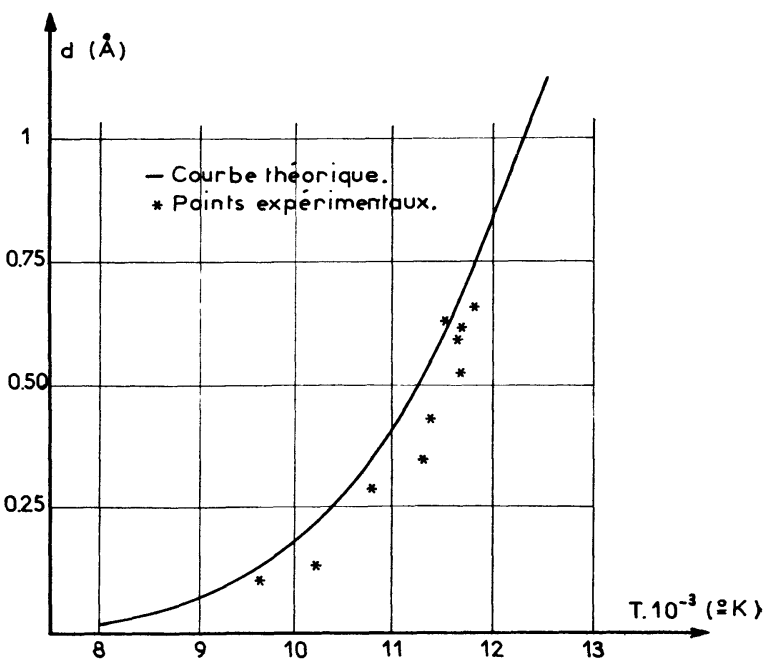

FIG. 8. - Déplacement de la raie $4300 \AA$ de Ar I.

$\mathrm{Au}$ voisinage de l'axe, l'accord entre l'expérience et la théorie de Griem est remarquable en ce qui concerne les demi-largeurs, et satisfaisant pour les déplacements. Les incertitudes sur la mesure des déplacements proviennent pour l'essentiel du repérage de la longueur d'onde $\lambda_{0}$ de la raie non perturbée 
(déterminée à $\pm 0,03 \AA$ près); une augmentation de l'intensité du courant d'arc, donc de la température, permettra d'étendre le domaine des mesures et d'obtenir une meilleure précision.

4. Etude expérimentale des profils des raies d'émission de la source $\mathrm{S}_{2}$. - 4.1 MESURE DE LA TEMPÉRATURE SUR L'AXE. - Nous avons cherché à voir dans quelle mesure la température est uniforme le long de l'axe du plasma. Pour cela, nous avons comparé la température obtenue par observation axiale (en bout) à celle existant sur l'axe aux trois points d'observation latérale, distants de $15 \mathrm{~mm}$. Si l'on considère que le plasma a une symétrie cylindrique parfaite, chaque couche concentrique a une température uniforme. Dans ce cas, l'observation, " en bout", du rayonnement émis par un cylindre de plasma de très faible diamètre et de même axe que l'arc, nous permet d'enregistrer non seulement le profil de la raie $\mathrm{H}_{\beta}$ dont la demi-largeur est directement liée à la concentration électronique, donc à la température, mais aussi les profils des raies de l'Ar I et de l'Ar II correspondent à la température axiale. Par contre, l'observation latérale, comme nous l'avons vu au $\S 3$, nécessite la transformation d'Abel pour obtenir la température axiale.

Nous avons étudié tout d'abord l'influence des fenêtres obstruant les ouvertures d'observation latérale. En utilisant le montage du § 3.3.2, nous avons enregistré plusieurs profils $I(x, \lambda)$ à longueur d'onde constante $\lambda_{i}$ : ces profils sont tous symétriques, et leur symétrie se conserve lorsqu'on enlève ou remet en place les fenêtres. Nous avons déduit que la symétrie du plasma est cylindrique et enlevé définitivement ces fenêtres latérales afin de rendre l'observation plus commode et éviter l'absorption partielle du rayonnement par le verre.

Les deux techniques d'enregistrement et de calcul (§ 3.3) que nous avons mises au point pour la détermination radiale de la température nous permettent d'obtenir la température axiale avec une erreur expérimentale variant, selon la technique, de $5 \%$ aux environs de $3 \%$. Ayant jugé cette précision insuffisante, nous avons renoncé à l'utilisation de la transformation d'Abel et procédé de la façon suivante : nous avons comparé la température obtenue par observation en bout, aux trois températures globales latérales correspondant aux demi-largeurs observées des profils de la raie $\mathrm{H}_{\beta}$ émise par une colonne de plasma passant par l'axe de l'arc par chacune des ouvertures d'observation latérale.

L'arc fonctionnant à la pression atmosphérique

$$
\begin{array}{cc}
\begin{array}{c}
\text { Températures } \\
\text { mesurées en bout } \\
\text { (moyennes) }
\end{array} & \\
- & \text { Côté Anode } \\
T_{0}=12600^{\circ} \mathrm{K} & T_{1}=12380^{\circ} \mathrm{K} \\
\Delta T_{0}=80^{\circ} \mathrm{K} & \Delta T_{1}=40^{\circ} \mathrm{K}
\end{array}
$$

dans les conditions suivantes : intensité du courant $77 \mathrm{~A}$, tension aux bornes de l'arc $97 \mathrm{~V}$, débit gazeux $10 \mathrm{l} / \mathrm{mn}$, et sans interruption, nous avons enregistré successivement plusieurs profils de $\mathrm{H}_{\beta}$ correspondant à chacune des quatre directions d'observation (une en bout et trois latérales).

Le tableau ci-dessous donne les moyennes des températures mesurées en bout et des températures globales latérales obtenues avec la dispersion des résultats notés $\Delta T$ pour chaque ouverture d'observation.

L'examen de ce tableau nous montre, d'une part, la bonne reproductibilité des résultats pour chaque observation et d'autre part, les valeurs très rapprochées de $T_{1}, T_{2}$ et $T_{3}$. Nous pouvons écrire $T_{4}=124000^{\circ} \mathrm{K} \simeq T_{1} \simeq T_{2} \simeq T_{3}$.

Si l'on observe le rayonnement global émis par un élément prismatique voisin d'un diamètre, à la raie large émise par les éléments paraxiaux plus chauds, se superposent des raies étroites émises par des éléments éloignés de l'axe qui ont pour effet de réduire la largeur apparente de l'ensemble : le mode d'observation latéral choisi donne une température globale inférieure à celle du centre du disque de plasma. Les résultats obtenus par Le Men [16] en utilisant la première technique $(\S 3.3 .1)$ et une source analogue à $S_{1}$ montrent que la température globale correspondant à la demi-largeur du profil $I_{x=0}(\lambda)$ de $\mathrm{H}_{\beta}$ est d'environ $300 \mathrm{~K}$ inférieure à la température axiale $\left(12150^{\circ} \mathrm{K}\right)$. Ceci montre que $T_{0}-T_{4}=200^{\circ} \mathrm{K}$ est supérieure à la différence entre $T_{0}$ d'une part, et chacune des trois températures existant sur l'axe aux points d'observation latérale. On en déduit avec une bonne approximation que, même si la température en ces trois points de l'axe est seulement supérieure à $T_{4}$ de $75^{\circ} \mathrm{K}$, l'erreur expérimentale commise sur la détermination de la température axiale par observation en bout, ainsi que sa variation le long de l'axe est de l'ordre de $1 \%$. Cette précision est du même ordre de grandeur que celle obtenue par d'autres auteurs [17] pour des plasmas de nature différente.

Nous noterons que même la plus avancée de nos deux techniques (§ 3.3.2) de détermination de la distribution radiale des températures d'un disque de plasma ne peut donner plus de renseignements concernant l'homogénéité de la température le long de l'axe.

4.2 Etude DU PROFIL Des RAIES DE L'AR I ET DE L'AR II PAR OBSERVATION AXIALE. - Dans les mêmes conditions de fonctionnement de l'arc que celles indiquées au paragraphe précédent, nous avons enregistré par observation axiale les profils de raies de l'Ar I

$$
\begin{array}{cc}
\begin{array}{c}
\text { Températures globales latérales } \\
\text { (moyennes) }
\end{array} & \\
\text { Centre } & \text { Côté Cathode } \\
T_{2}=\overline{12} 415{ }^{\circ} \mathrm{K} & T_{3}=\overline{12} 395{ }^{\circ} \mathrm{K} \\
\Delta T_{2}=30 \circ \mathrm{K} & \Delta T_{3}=70 \circ \mathrm{K}
\end{array}
$$


et de l'Ar II. Nous nous sommes particulièrement intéressés à la raie $4158 \AA$ de l'Ar I et aux raies $4806 \AA$ et $4426 \AA$ de l'Ar II. Les largeurs des fentes d'entrée et de sortie du monochromateur sont fixées à $20 \mu$.

Sur la figure 9, qui représente le profil expérimental de la raie $4158 \AA$, pour laquelle le plasma peut être considéré comme optiquement mince [18], nous avons tracé le profil de dispersion, de même intensité maximum et passant par le point $M$ du profil de la raie (point situé à $I$ max $/ 2$ du côté des faibles longueurs d'ondes). Si l'on tient compte de la contribution de l'intensité de la raie $4164 \AA$ de l'Ar I du côté des grandes longueurs d'ondes, nous pouvons considérer avec une bonne approximation que le profil de la raie 4158 de l'Ar I émise par une colonne de plasma à température uniforme est un profil de dispersion.

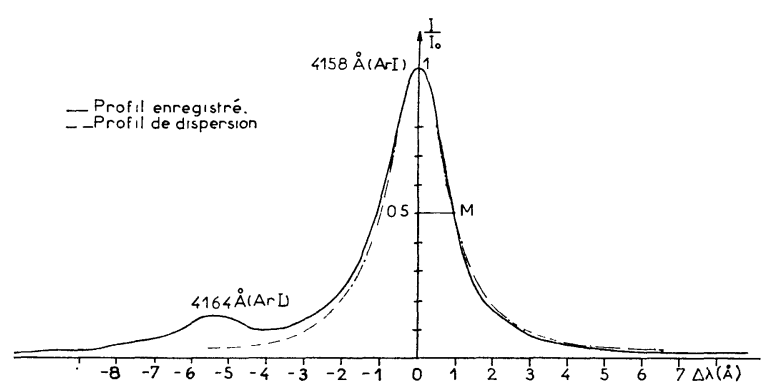

FIg. 9. - Profil des raies $4158 \AA$ et $4164 \AA$ de Ar I enregistré par observation axiale.

Sur les figures 10 et 11 , nous comparons les profils expérimentaux des raies $4426 \AA$ et $4806 \AA$ de l'Ar II aux profils de dispersion de même demi-largeur. Ces deux raies, pour lesquelles nous avons considéré que le plasma est optiquement mince, ont un profil de dispersion, comme l'ont déjà montré d'autres auteurs [19] en utilisant des techniques et des sources de plasma différentes.

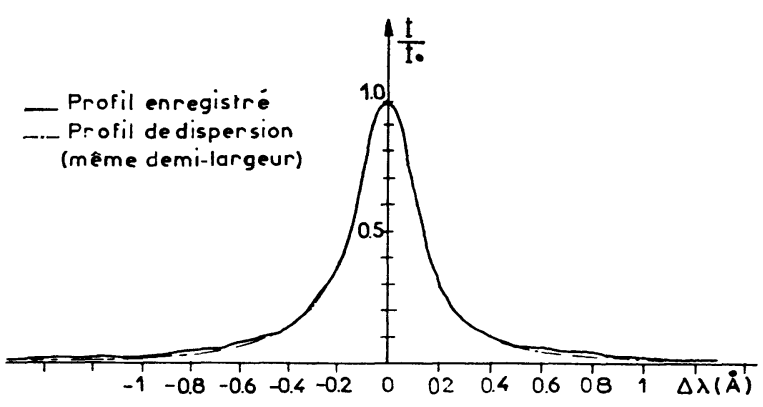

Fig. 10. - Profil de la raie $4426 \AA$ de Ar II enregistré par observation axiale.

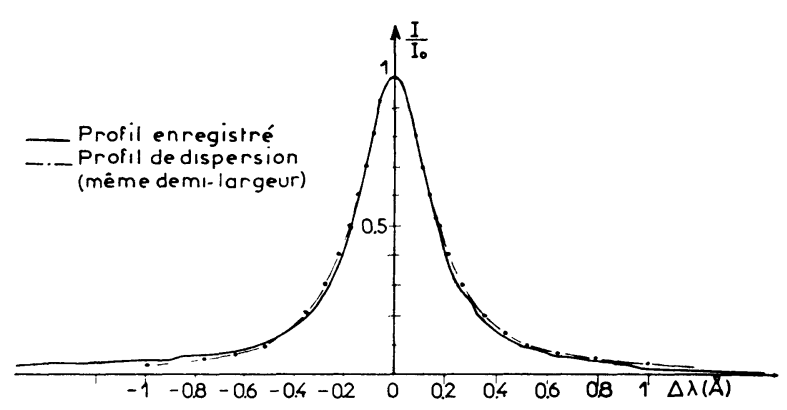

FIg. 11. - Profil de la raie $4806 \AA$ de Ar II enregistré par observation axiale.

5. Conclusion. - Nous avons réalisé deux sources de plasma stables fonctionnant dans l'argon ou dans un mélange d'argon et d'hydrogène, et mis au point quelques techniques propres à l'étude de l'élargissement et du déplacement des raies d'émission d'un plasma à symétrie cylindrique par observation latérale.

L'arc $S_{2}$ présente une température pratiquement uniforme le long de l'axe. Il permet, par l'observation axiale du plasma, d'obtenir directement sans correction ni transformation, le profil et l'élargissement des raies émises par des éléments du plasma situés sur l'axe.

On peut donc, avec cet arc, mesurer directement l'élargissement des raies de Ar I et de Ar II, ou de la raie $H_{\beta}$ de l'hydrogène, donc de la température sur l'axe.

\section{Bibliographie}

[1] Quefrelec (J. L.), Thèse 3e Cycle, Rennes, 1963.

[2] Maecker (H.), Z. Naturforsch., 1956, 11a, 457.

[3] ShumaKer (J. B.), The Review of Scientific Instruments, 1961, 32, 1, 65-67.

[4] Wiese (W. L.), Paquette (D. R.) and Solarski (J.E.), Phys. Rev. Letters, 1963, 129, 3, 1225-1232.

[5] Finkelnburg (W.) and MaeCKer (H.), Handbuch der Physik (Springer-Verlag, Berlin, 1956), Vol. 22.

[6] Lochte Holtgreven (W.), Production and measurement of hight temperatures-Reports on progress in physics, Vol. 21, 312, 1958.

[7] Shumaker (J. B. jr) and Wiese (W. L.), Temperature. Its measurement and control in Science and Industry. Vol. 3, Pt 1, pp. 575-579, 1962.

[8] Griem (H. R.), Plasma spectroscopy, Mc Graw Hill, New York, 1964.

[9] Saha (M. N.), Phil. Mag. J. Sc., 1920, 40, 472 et 809; 1921, 41, 267. Proc. Roy. Soc. A, 1921, 99, 135. Z. S. Physik, 1921, 40, 6.
[10] Eggert (J.), Phys. Z. S., 1919, 20, 570.

[11] Margeneau (H.) et Lewis (M.), Rev. Mod. Phys. $1959,31,569$.

[12] Griem (H. R.), Phys. Rev., 1962, 128, 997.

[13] Drawin (H. W.) et FelenboK (P.), Data for plasmas in local thermodynamic equilibrium, GauthierVillars, Paris, 1965.

[14] Legras (J.), Précis d'Analyse numérique, Dunod, Paris, 1963.

[15] Cabannes (F.) et Sy (A.), J. Rech. du C. N. R. S., $1962,58,37$.

[16] Le Men (R.), D. E. S., Rennes, 1967.

[17] Wiese (W. L.) and MURPhy (P. W.), Phys. Rev., 1963, 131, 2108-2115.

[18] Chapelle (J.), Sy (A.), Cabannes (F.) et Blandin (J.), J. Quant. Spectrosc. Radiat. Transfer., 1968, 8, 1201-1216.

[19] Chapelle (J.), Sy (A.), Cabannes (F.) et Blandin (J.), C. R. Acad. Sci. Paris, 1968, 266, 1513-1516. 